\title{
Supply Chain Modeling Based on Restructuring Activities
}

\author{
Lucian Hancu \\ "Babeş-Bolyai" University and SoftProEuro s.r.l. \\ Romania
}

\section{Introduction}

The restructuring activities of the type Mergers and Acquisitions (M\&A) constitute a real challenge for the companies competing with the ones involved in M\&A processes, as such a restructuring process usually improves the resulting company's position on the market by creating or consolidating a competitive advantage. The competitive advantages take the form of reducing overall costs by economies of scale, access to new markets, access to new products or technologies.

From the perspective of Supply Chain Management, the Mergers and Acquisitions can induce changes in the supply chain, which affect the life of business entities, especially the small ones, which remain unable to quickly adapt to these changes; thus struggling to survive in the turbulent business environment.

Current Supply Chain Management techniques mostly rely on the barcodes and Radio Frequency Identification (RFID) technologies in order to keep track of the product from its assembly line to the final customer. A noted example of large-scale RFID usage is WalMart's request that its top one hundred supplier introduce RFID tags for better tracking their products, distribute these products more efficiently and eliminate abnormal costs from the shipment and distribution to Wal-Mart (Sliwa, 2004), cited in (Fuld, 2006) and (Hancu, 2008b).

Although there are clear advantages of introducing such technologies in the product's lifecycle, small entities might encounter difficulties (for instance, lack of financing) in implementing such technologies on a larger scale, which limits the applicability of the mentioned technologies in revealing the changes in the supply chains due to the Mergers and Acquisitions activities.

On the other part, accessing data regarding product tracking from the assembly line to the customer constitutes a real challenge. Such data remains private to the companies involved in the supply chains and is accessible only through authentication interfaces or private tracking identification numbers (as in the case of Web-based package tracking systems provided by most courier companies).

In this chapter, we describe a different approach in studying the changes in the supply chains due to mergers and acquisitions activities, based on constructing a set of Virtualized Supply Chains (VSCs) and applying the mergers-induced changes to these Virtualized 
Supply Chains. Our aim is to reduce the risks in the supply chain by highlighting which one of the three investigated mergers alternatives (upstream vertical merger, downstream vertical merger and conglomerate merger) is better suited for diminishing the risks in the supply chains sets.

In order to quantify the implications of the three types of mergers to the supply chain, we first generate a list of Virtualized Supply Chains and then we compute the risks associated with each supply chain and the risks corresponding to each business class that appears as a member of the Virtualized Supply Chain. We apply the selected mergers strategies, compute the risks associated with the new Virtualized Supply Chains; then compare the initial risks with the ones highlighted after introducing the mergers shifts in the supply chain.

The chapter is structured as follows. In the next section, we provide a survey on the research literature correlated to the Supply Chain Management (SCM) field the measuring and the managing of risks in the SCM. The third section introduces the terminology that we later employ during our research, which regards the virtual modeling of the Supply Chains: business class, business dependency, bounded and unbounded Virtualized Supply Chain, risk of a business class and global risk of the Virtualized Supply Chain Set.

The subsequent section describes our method of modeling Virtualized Supply Chains by applying the three restructuring strategies: upwards vertical merger, downwards vertical merger and conglomerate merger. The fifth section of the chapter presents the results of our modeling, while the subsequent section highlights our final conclusions.

\section{Related work}

Studying the impact of the mergers activities on the supply chains is a noted subject of academic research, as mergers usually consist in integrating the operations of the involved companies, from the step of introducing an order, purchasing raw materials, processing operations, the financial aspects of both orders to suppliers and requests from clients, warehouse operations when required and final shipment of the products to the clients (DePamphilis, 2009).

Vertical mergers are combinations of entities along the supply chains with a great chance of improving economic efficiency by cutting intermediaries and reducing overhead (Harrigan, 1985), cited by (Bruner, 2004). An example of vertical merger would be the merger between an aluminium smelter and a bauxite miner (upstream vertical merger) or between the same aluminium smelter and an airplane producer (downstream vertical merger). As mentioned in (Bruner, 2004), supply chain disruptions are listed among the classic concerns of operating managers, based on the fact that a merger or alliance between two companies has great impact among both clients and suppliers. This was also the case with the recent alliance between two IT distributors from Romania (Preda, 2009), which conducted to the appearance of a larger competitor in the IT distribution market. Even if a full integration and merger is far from being completed, involving anti-trust laws, competitors tend to be seriously affected by such a merger or strategic alliance.

Various forms of collaborations in the Supply Chains are enlisted in (Meca et. al., 2008). The research is conducted on the principle of multi-firm analysis that has become the subject of research in the operations management field, due to the market globalization and a more intense competition among business entities. The article distinguishes between horizontal (cooperation between peers, in the form of inventory cooperation) and vertical cooperation through the supply chain. The vertical coordination is divided into the coordination of 
actions and a full merger, in the first case the two actors act independently, while in the case of the mergers the parties are under centralized control and give up their independence as now constitute a single business entity. According to the authors, there is a limited research studying cooperative game theory as the base for vertical cooperation in the Supply Chain. In our opinion, the distinction between vertical coordination as two independent companies and full merger cooperation should not be treated separately, from the Supply Chain perspective. We have seen in practice that a full integration is realized after a long process of integration of the two business entities, as it was the case of the two IT distributors, described in (Preda, 2009).

A framework for ranging business entities through the Supply Chain is proposed by (Huo, 2009). According to the performance of the suppliers, the business entities would be classified into partners, key suppliers, qualified suppliers and in questioned suppliers. The partners qualification is given to the top performance suppliers, key suppliers to the ones that have reached the minimum threshold performance and are going to the top class, qualified suppliers for the ones that have reached the minimum requirements but no improved action has been observed, while the in question suppliers are the ones that have never reached the minimum requirements and are expected to be rejected from being suppliers. A system for evaluation of the suppliers is also given in the article, each supplier being inspected in terms of Quality, Delivery, Technical support, Collaboration and Cost. The Partner certification is given to suppliers obtaining a score between $92 \%-100 \%$, Key suppliers to the ones ranging between 80\%-91\%, Qualified supplier: 70\%-79\% and In questioned suppliers 70\%.

Our approach in managing risks through the supply chain is based on a similar principle that the one highlighted in (Huo, 2009), with the difference that we categorize classes of business entities instead of single business entities. That is, we would rank as high risk a whole business class instead of a single company. The reason for applying such approach is that the same risk category can be assigned to the whole business class, as there would be a higher probability that prices would increase in that business class, which would induce a risk in the supply chain by altering the costs of that business class's primer consumers, then propagating such risk through the supply chain. We highlight our approach of classifying business classes according to the risk measures in the next and fourth section of the chapter.

A thorough analysis of supply chain risks is provided in (Kiser et al., 2006). According to the authors, external risks can be revealed by facts that appear either upstream or downstream through the supply chain. These risks are:

- Demand risks - the customer demand is unpredictable or misunderstood.

- Supply flow risks - interruptions than might appear in the flow of the product.

- Environment risks - shocks that appear outside of the supply chain, but significantly influence the supply chain (e.g. the rising of oil prices due to the starting of an army conflict in one of the oil export countries).

- Business (or Financial) risks which express the financial or managerial stability of the supplier

- Physical risks which correspond to a change in the conditions of the physical facilities of the supplier.

In contrast, internal risks are fired by the events that occur within the company:

- Manufacturing risk caused by changes in the internal processes or operations

- Business risks - when modifications are made in key personnel, management personnel or the reporting departments. 
- Planning and control risks - ineffective management or a planning process that is not appropriate

- Mitigation and contingencies - not putting contingencies in place.

The authors (Huo et al., 2006) propose a 6 step process of risk management within the supply chain, the steps involved being:

- Supplier base: identifying each row material that is needed in producing the desired result, identifying the strategic materials and understanding suppliers' organization.

- Vulnerability: the business entity should reveal the scenarios that are likely to happen, identify the causes that would imply them to appear, and the measure in which the company is more or less able to cope with these scenarios.

- Implications: use simulations in order to quantify the impact of each risk when appeared

- Mitigation: when an unexpected event occurs, how the company should act in order to get back to a normal situation and continue its activity in a natural way.

- Costs and benefits: costs in mitigation actions are accompanied by a benefit from reducing risks and lowering costs when a risky event appears.

- Measures and actions: identifying roles and responsibilities within the organization for partnering with suppliers in order to secure the supply chain. The idea is similar with the one expressed in (Hancu, 2011), in which a mechanism of replacing an abnormal unavailability of a supplier by one of its peers is proposed.

Analyzing risks from a company's perspective conducts to a more thorough analysis compared with the case of analyzing classes of business entities. Despite this lower-accuracy inconvenient of analyzing classes of business entities, the business classes approach has the advantage of scale, as its conclusions are applicable to a larger number of business entities than in the case of a single analysis. With the aiming of a higher impact advantage, we present a method of reducing risks in virtually constructed Supply Chains based on restructuring activities of various mergers types. The following section introduces the main definitions employed in our research, which we shall later use during our methodology description.

\section{Definitions}

In this section, we describe the terminology that we later apply in our research. Instead of tracking products by one of the techniques that the research literature highlights, we generate a set of Virtualized Supply Chains, which are computed by linking two classes of business entities that come in a direct dependency relation.

The Virtualized Supply Chains link various direct-dependent business classes, thus dealing with a greater diversity of Supply Chains examples than in the case of tracking products via Radio Frequency Identification (RFID) or barcode scanning. The later Supply Chain tracking technique would be limited to the business entities that use barcodes or RFID, usually formed by courier companies or large super markets like Wal-Mart.

\subsection{The class of a business entity}

The class of a business entity denotes its main activity code according to a standard classification scheme. In our research, we use the Romanian Classification of the Economic Activities (CAEN), which complies with the European Standard of Classification of the Economies (NACE). 


\subsection{Business dependency}

We denote that two business classes Class $\mathrm{A}$ and Class $\mathrm{B}_{\mathrm{B}}$ come in a Business Dependency relation, when two or more Business entities (one having its main activity code Class ${ }_{A}$ and the other - Class $_{\mathrm{B}}$ ) establish relationships of the type supplier-client. By gathering all the business dependencies from the Business classes of the analyzed entities, a Business Dependency Map can be computed, which is later employed in the construction of the Supply Chains.

The Business Dependency Map establishes relationships between classes of business entities and the probabilities that various business classes depend on other business classes. We have previously constructed the Business Dependency Map from the online search logs of our Web-based search application for business entities, by considering a dependency between the first searched entity's business class and the following searched entities' business classes coming from the same Web session (Hancu, 2008a).

\subsection{Bounded and unbounded virtualized supply chains}

A Virtualized Supply Chain (VSC) is a list of business classes that come in a direct dependency relation. The Virtualized Supply Chains are constructed as follows. Instead of considering as parts of the supply chain a specified producer, its suppliers and clients, we construct a Virtualized Supply Chain by linking classes of business entities (for instance: Restaurants and Production of bread) that come in a direct dependency relation according to the Business Dependency Map.

The Virtualized Supply Chains can be either unbounded, as introduced in (Hancu, 2008b), or bounded. An unbounded VSC denotes a supply chain which is constructed by linking directdependent business classes until no more business classes can be added at the end of the last business class, as there is no other business class available for the insertion (i.e. no class which depends on the last-added Business Class and which has not already been added to the VSC).

In this chapter, we introduce the notion of Bounded Virtualized Supply Chain, which denotes a Virtualized Supply Chain that has the length $n$, i.e. it links exactly $n$ directdependent business classes. The introduction of Bounded Virtualized Supply Chains comes as a step forward in the study of Virtualized Supply Chains and it enhances the studies from our previous work (Hancu, 2008b).

Considering that the Business Classes Restaurants and Production of bread are in a dependency relation (which means that a business entity from the Restaurants business class can have a client or supplier an entity of the Production of bread business class), we can construct a Virtualized Supply Chain that starts with either the two business classes, then are followed by other business classes that come in a direct dependency relation with the last business class from the supply chain.

The bounded Virtualized Supply Chains are the supply chains that are of a specified size (for instance, the 5-Bounded Virtualized Supply Chains are the VSCs that are formed of five business classes that are each one dependent with the previous business class according to the Business Dependency Map).

The unbounded Virtualized Supply Chains are the supply chains constructed from a starting business class that have no possibility of expanding, i.e. no other business class can be added at the end of the Virtualized Supply Chain that comes in a direct dependency with the last-added business class of the Virtualized Supply Chain (as the last-inserted business class has no direct dependants, or its direct dependant business classes were previously added to the Virtualized Supply Chain). 


\subsection{Risk of a business class, global risk of the virtualized supply chain set}

By considering the computed set of both bounded and unbounded Virtualized Supply Chains, we calculate the risk of each business class by flooding each Virtualized Supply Chain with a starting risk of 10 units (which means that the company from that business class can increase prices by $10 \%$ or can lower its production by $10 \%$ ).

We propagate the risk through the Virtualized Supply Chain and sum up all the risks associated with each business class, then classify the business classes as High-risk business classes, Medium-risk business classes and Low-risk business classes.

The Risk of a Business Class sums all the risks associated with that business class, after the flooding of the risks in the set of Virtualized Supply Chains. Instead, the Global Risk associated to the Virtual Supply Chain Set sums all the risks from all the Business Classes. The goal of our research is to reduce the Global Risk by applying merger strategies. The methodology is further discussed in the next section of this chapter.

\section{Research method}

The construction of the Business Dependency Map explained in (Hancu, 2008a) yields to the derivation of a list of probabilities between pairs of business classes, that we can employ in the construction of Virtualized Supply Chains, both bounded and unbounded, as explained in the previous section. For the initial set of Virtualized Supply Chains, we compute the global risk by flooding each VSC with an initial risk of 10 units, than adding to the current risk the previously computed supply chain risk. The current risk is formed by the probability that the current business class depends on the previous business class in the VSC.

Our previous approach highlighted in (Hancu, 2008b) employed products instead of sums in the computation of the current risk. The current risk was calculated as a product between the past risk and the probability that the current business class depends on the previous business class. In a thorough analysis of our past approach, we have realized that the current risk becomes insignificant, as the probability of correlated business classes is usually less than $10 \%$, in some cases being even much smaller.

While the past approach made the assumption that the risk decreases with the browsing of the Virtualized Supply Chain (see Figure 1 for an example of such a 5-bounded Virtualized Supply Chain), we now assume that such risk increases while we navigate through the Virtualized Supply Chain.

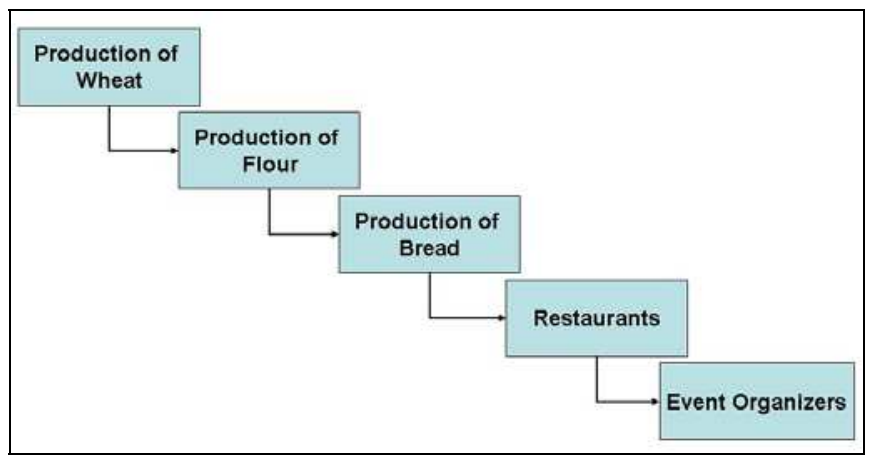

Fig. 1. Example of a virtualized supply chain 
With the purpose of reducing the risks of the supply chains, we compare the results of applying three merger strategies to the Virtualized Supply Chains: upstream vertical merger (an example is depicted in Figure 2), downstream vertical merger (see Figure 3 for an illustrated example) and conglomerate merger (highlighted in Figure 4).

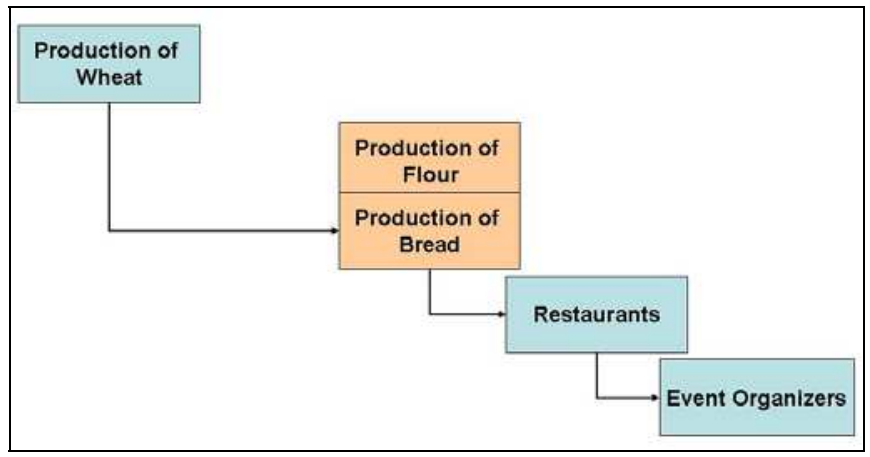

Fig. 2. Example of an upstream vertical merger

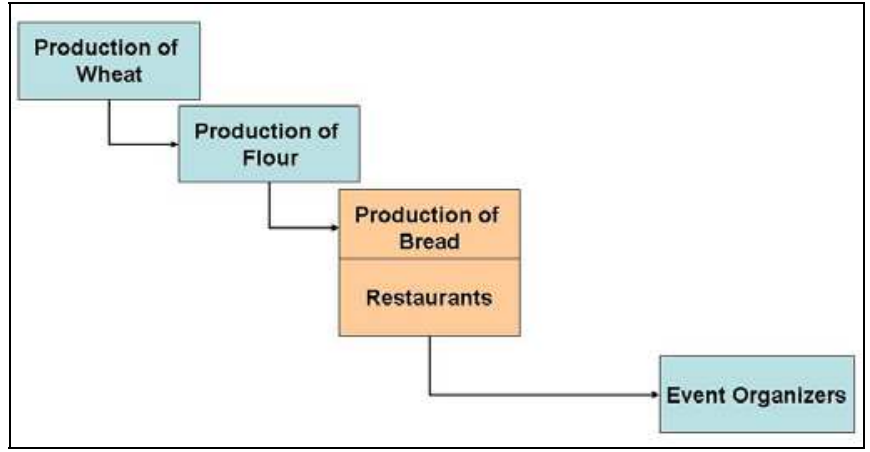

Fig. 3. Example of a downstream vertical merger

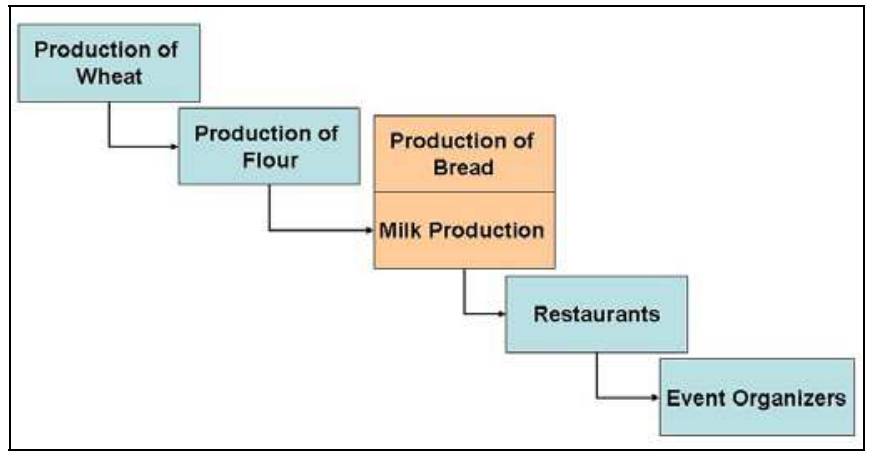

Fig. 4. Example of a conglomerate merger 
The three mergers strategies and their induced changes to the supply chains are highlighted in the above-mentioned figures together with the initial unaltered Virtualized Supply Chain. The upstream vertical merger proposes a merger between the Production of flour and Production of bread business classes (we would suggest that a company whose business activity code is Production of flour should merge with a company whose activity code is Production of bread); the downstream vertical merger suggests a strategic action between the Production of Bread and Restaurants business classes, while the conglomerate merger strategy would suggest a merger between two different business classes that are neither upstream nor downstream vertical mergers (they are not closely related in the supply chain).

In contrast with our previous studies, in which we have applied only a risk-reduction technique similar to the conglomerate merger one (that consisted in replacing the high-risk business classes with medium-risk business classes, then replacing high- and medium-risk business classes with low-risk business classes), we now apply three different risk reduction strategies. The three strategies (upstream vertical mergers, downstream vertical mergers and conglomerate mergers) consist in replacing the business classes from the high-risk business classes with its upstream (respectively, downstream) lower business classes from the supply chain, together with replacing high-risk business classes with unrelated business classes (that are neither upstream, nor downstream close in the Virtualized Supply Chain in the case of conglomerate mergers) which exhibit lower risks. In the next section of the chapter, we highlight the results of our experiments together with our findings regarding the risk reduction strategies in the Virtualized Supply Chains by applying the three different types of mergers as restructuring activities.

Although the risk reduction technique is similar with the one presented in (Hancu, 2008b), there are some differences in our current approach with respect to the one of our previous studies. The first and foremost difference relies from the computation of the risk associated with each Virtualized Supply Chain, thus having a significant impact on the final computation of the Global Risk of the VSC set.

We pointed out that in our previous approach we have used products for linking the previous risk of the business class with the current risk of the business class in the VSC. This conducts to the diminishing of the current risks of the business classes, as we navigate through the Supply Chain. As the algorithm of the unbounded VSC construction yields to longer Virtualized Supply Chains (formed by up to 251 business classes), and the dependencies probabilities less than 0.10 , the risk soon becomes insignificant. Summing insignificant risks yields to the computation of a lower global risk, which was the case of our results from our past researches.

On the other part, the risk reduction algorithm functioned on a set of Virtualized Supply Chains which were almost zero, in the case of our previous approach. This implied that the risk reductions were made at the beginning of the Virtualized Supply Chain, where the risks were higher, thus contributing to a lowering of the overall Global Risk associated with the Virtualized Supply Chains set. In the subsequent section, we depict the results from our research and discuss our findings.

\section{Applying restructuring strategies to supply chains}

Our experiments are generated as follows. We compute the set of the Virtualized Supply Chains and then calculate the global risk of the VSC, which conducts to the computation of the list of High Business Class, Medium Business Class and Lower Business class. We later apply the conglomerate merger strategy by replacing high-risk business classes with 
medium and low risk business classes. In the subsequent step, we apply both strategies for Upstream and Downstream Vertical merger in the same time.

The upstream and downstream vertical mergers occur as follows: first, we identify a business class $\mathrm{A}$ and consider the classes $\operatorname{Prev}_{\mathrm{A}}$ and $\mathrm{Next}_{\mathrm{A}}$ (as the previous and next classes in the supply chain). If the risk of the business class $\operatorname{Prev}_{\mathrm{A}}$ is high and there is a dependency probability between the previous class (the Upstream Business class) of Prev $_{A}$ and A, we can apply the Upstream Vertical Merger to Prev ${ }_{\mathrm{A}}$ and A. If the risk of the business class $\mathrm{Next}_{\mathrm{A}}$ is high and there is a dependency probability between the A and the subsequent class (the Downstream Business class) of $\mathrm{Next}_{\mathrm{A}}$, then we can apply the Downstream Vertical Merger to Prev $_{A}$ and A. In the case we can apply both Upstream and Downstream Vertical mergers at the same business class $A$, we construct the two new supply chains, for each of the applied strategies.

The reason for applying both upstream and downstream vertical mergers during the same experiments is the following: a downstream vertical merger at the $n$-th step in the VSC would be equivalent with an upstream vertical merger at the $n+1$-th step. Processing Upstream and Downstream experiments in two different steps would imply that some of the upstream mergers in the first experiment would be equivalent to the downstream mergers in the second experiment.

We repeat the same mergers-based risk reduction strategies for both unbounded Virtualized Supply Chains and Bounded Virtualized Supply Chain with threshold above or equal to 5. The Unbounded Virtualized Supply Chain construction computes the maximal length of a VSC as 255.

The results are highlighted in the following figures. In the Figure 5, we depict the whole experiments: no risk management strategy, conglomerate merger, upstream (upward) vertical merger and downstream (downward) vertical merger. We notice a different trend of the Conglomerate risk management strategy with respect with the other management strategies, which follow the same pattern.

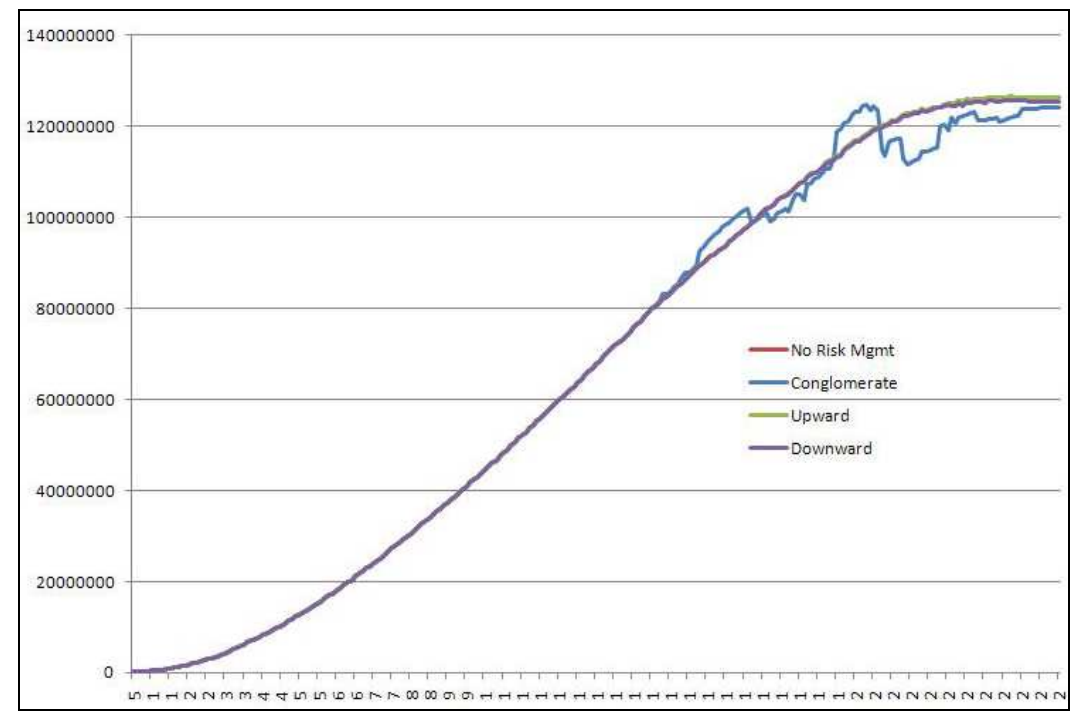

Fig. 5. Risks for 5-bounded VSCs to unbounded VSCs 
In the Figure 6 and Figure 7 we depict the experiments from the bounded Virtualized Supply Chains, with the aim of revealing the differences between the risk management strategies. The shapes reveal that there is not a significant difference between the no risk management and upward vertical merger, while Conglomerate merger is the best risk management strategy for VSC starting the 207 threshold.

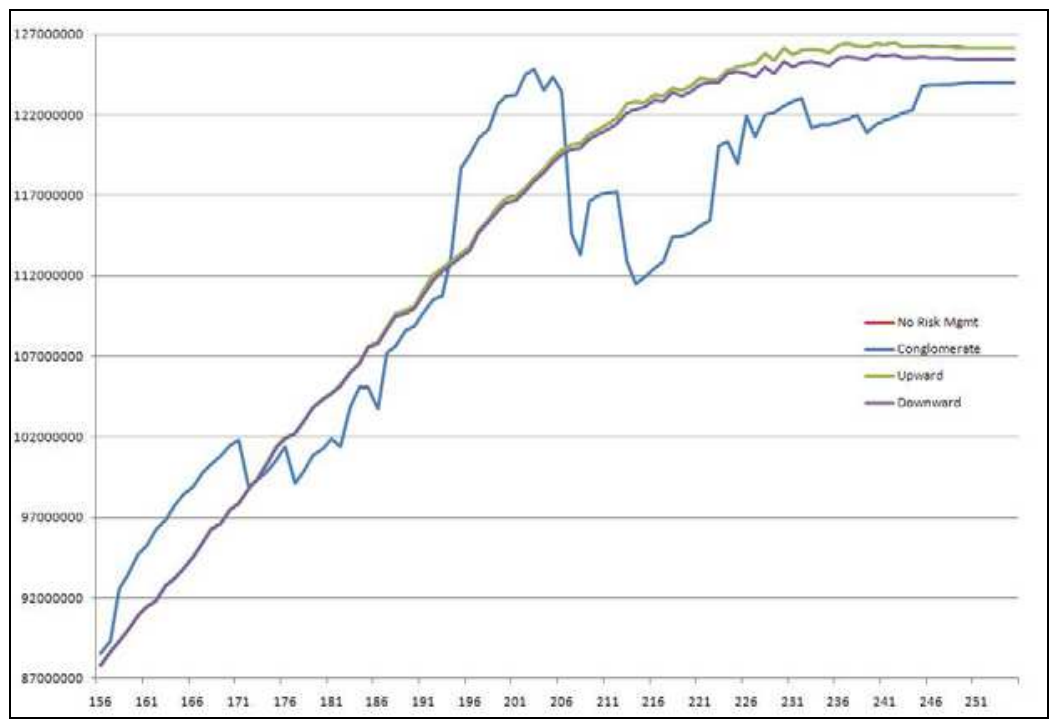

Fig. 6. Risks for 156-bounded VSCs to unbounded VSCs

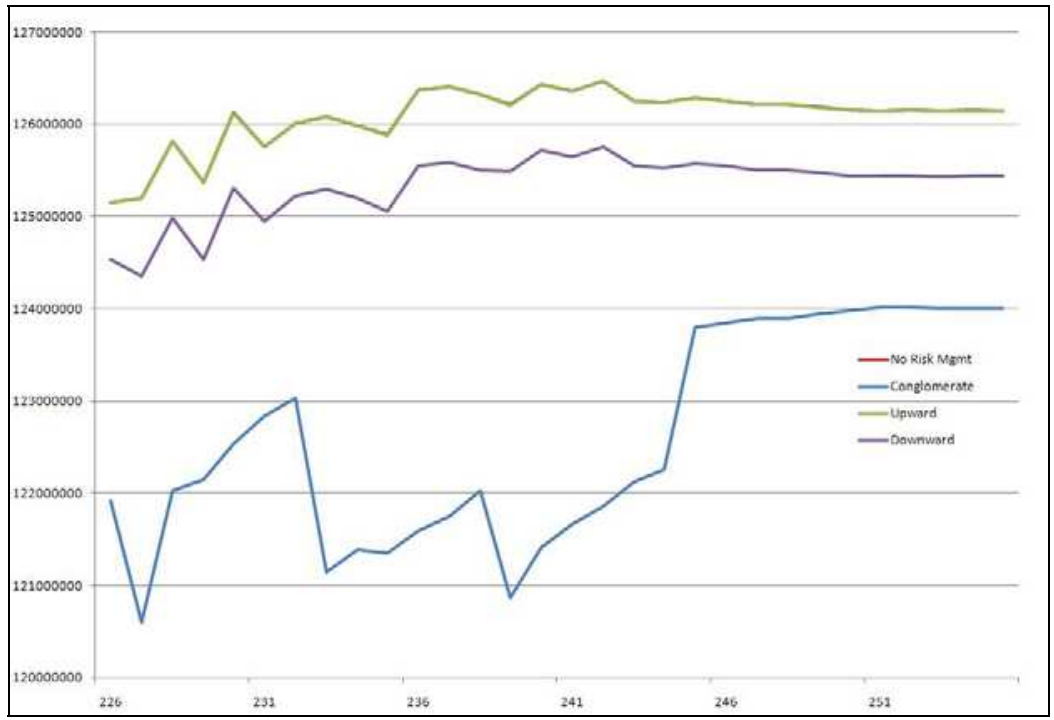

Fig. 7. Risks for 227-bounded VSCs to unbounded VSCs 
A more thorough analysis is done in the Figure 8 and Figure 9, where we depict the difference between Conglomerate and Downwards Vertical merger and between No risk management and Upwards Vertical merger. The analysis points out that the upwards risk management is better than applying no risk management, while conglomerate mergers are better suited for reducing risks than downstream vertical mergers.

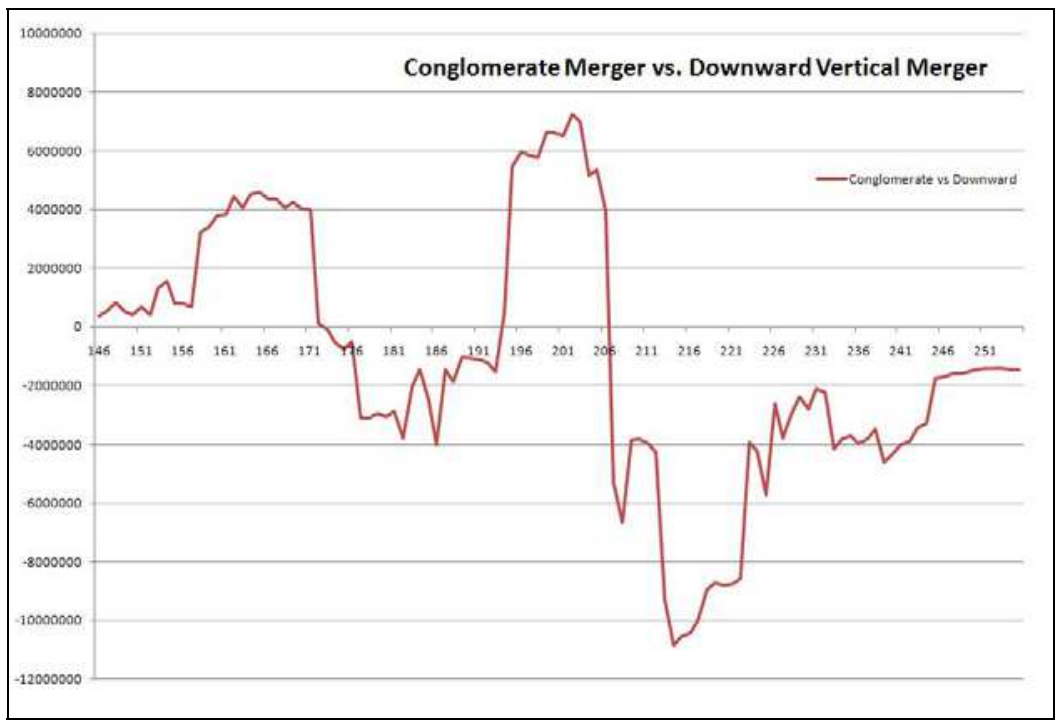

Fig. 8. Conglomerate compared to downward vertical merger risks

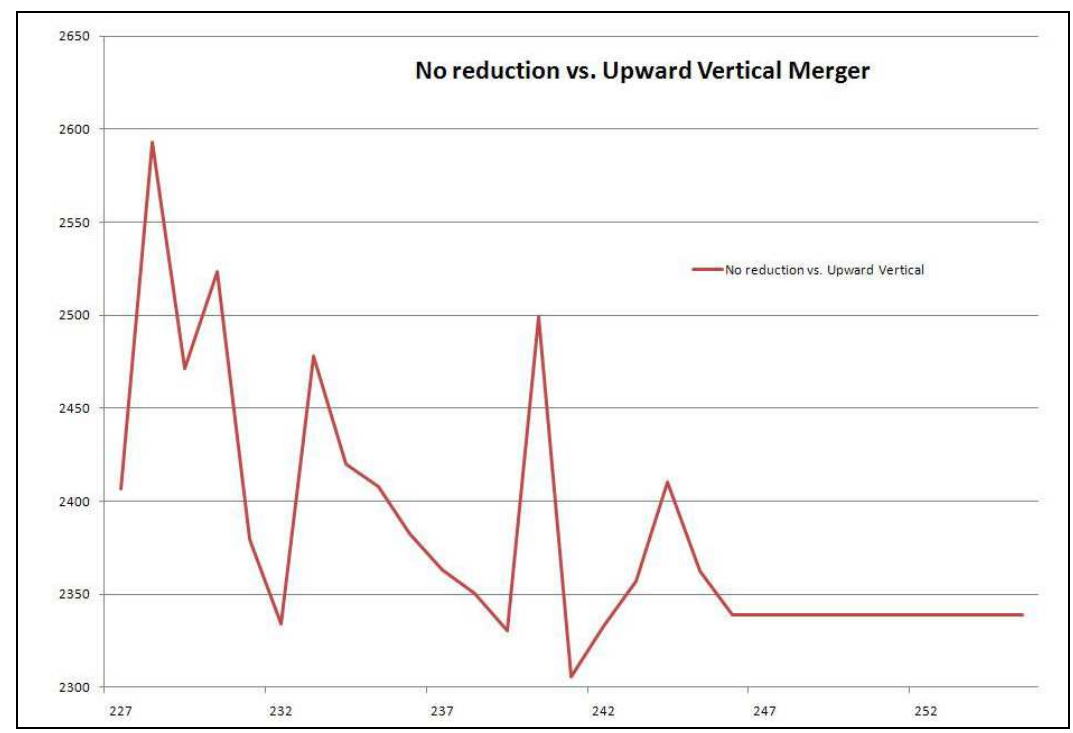

Fig. 9. No reductions compared to upward vertical merger risks 


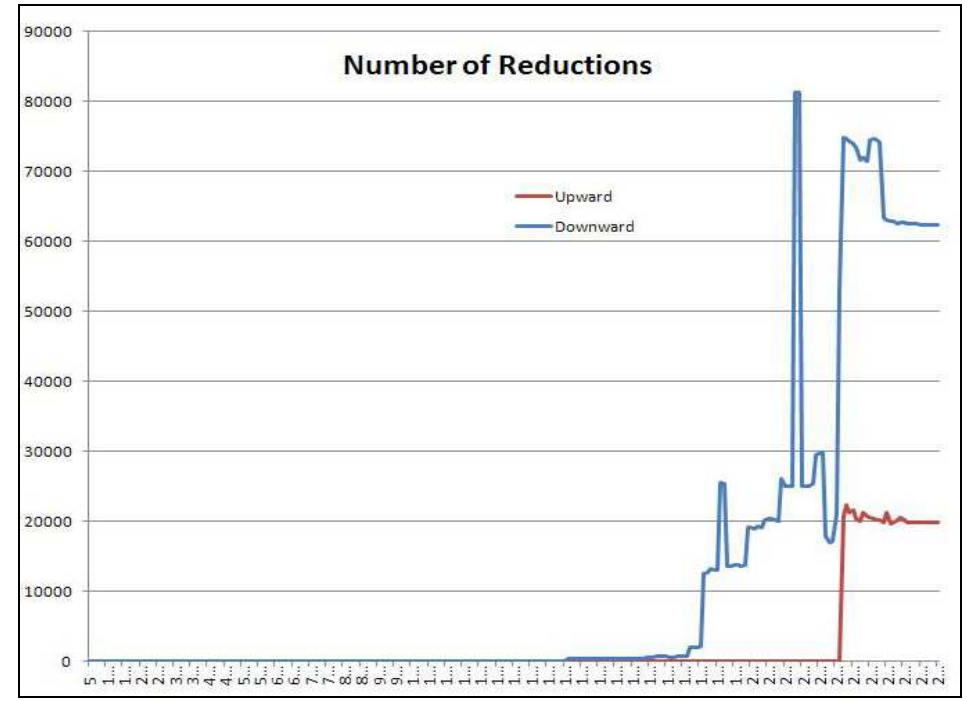

Fig. 10. Number of reductions for upward and downward vertical mergers

In the Figure 10, we have depicted the number of reductions from Upward and Downward vertical mergers, with the sole detail that the number of upwards vertical mergers reductions is multiplied by 100 , as to appear in the Figure. The figure highlights that there is a threshold above which the risk reductions appear. We denote as risk reduction a replacement of a higher-risk business class by a lower-risk business class, by one of the strategies pointed out above. The threshold is lower for Downward business classes with comparison with the upwards business classes and the number of risk reduction is also significantly higher. This conducts to the fact that the downstream vertical merger is better suited for risk management than the upstream vertical merger. Obtaining more risk reductions for downstream vertical mergers and a lower threshold for the beginning of risk reductions are the causes for which downstream vertical mergers are less risky than the other ones, according to our risk computation algorithm.

As highlighted in the Figure 10, there are VSC for which there are no reductions, which yields to the computation of the same global risk of the VSC set in the case of no-riskreduction, upwards and downwards vertical mergers.

An interesting fact appears as we compute the global risks for the conglomerate mergers risk reduction strategy. The Figures 5, 6, and 8 reveal that there are VSC sets for which the global risk is higher in the case of applying the conglomerate strategies than in the other strategies, while for VSCs starting the 207 threshold, the conglomerate merger is best suited for reducing risks (see also the excerpts depicted in the Table 1).

The results from our approach significantly differ from the ones depicted during our past researches, as we have introduced a slightly different method in the computation of the global risk of the VSC set and the risks of all of its associated business classes. Instead of multiplying the current dependency probability with the previous risk, we sum the current dependency probability with the previous risk. This yields to higher global risks associated with the business classes, while the reductions of the risks is lower, as compared with the results from our previous experiments highlighted in (Hancu, 2008b). 


\begin{tabular}{|c|c|c|c|c|}
\hline Threshold & No risk mgmt & Conglomerate & Upstream & Downstream \\
\hline 207 & 120126931,1 & 114579004 & 120126931,1 & 119865803,9 \\
\hline 208 & 120224021,6 & 113318490,3 & 120224021,6 & 119964766,8 \\
\hline 209 & 120784228,6 & 116613522,8 & 120784228,6 & 120462697,3 \\
\hline 210 & 121135881,3 & 117000408,7 & 121135881,3 & 120829932,6 \\
\hline 211 & 121432329,5 & 117164522,5 & 121432329,5 & 121125697,1 \\
\hline 212 & 121771115,3 & 117206781,3 & 121771115,3 & 121464914,7 \\
\hline 213 & 122679136,7 & 112904153,1 & 122679136,7 & 122171811,2 \\
\hline 214 & 122836452,1 & 111465331,5 & 122836452,1 & 122327477,7 \\
\hline 215 & 122778063,5 & 111939860,8 & 122778063,5 & 122468845,7 \\
\hline 216 & 123229506,3 & 112486049,9 & 123229506,3 & 122919765 \\
\hline
\end{tabular}

Table 1. Global Risk score (excerpts)

The insights derived from our research reveal that the downward vertical mergers are less risky than the upward vertical ones, and conglomerate mergers are the less risky approach in the Virtualized Supply Chains that are above the 207 threshold. We explain our findings as follows: merging with a distributor is less risky than merging with a supplier. While the supplier might increase its prices and alter our profitability by unexpectedly increasing production costs, the distributor's pressure (or power of negotiation) should be significantly lower. In addition, the supplier could have an increased position on the market, which could limit our options of substituting him with one of its competitors. In contrast, the distributor's powers should be lower, as we could always choose an increased number of smaller distributors instead of a larger one, thus this larger distributor would not obtain a significant negotiation power.

On the other part, a conglomerate merger should increase product diversification and that would imply lower risks. For instance, a conglomerate between an aluminium smelter and a steel producer would not be as affected by the increase of the bauxite price as a sole aluminium smelter alone. There are also cases in which the risk associated with the conglomerate merger is greater than the one in the case of no-risk management strategy or the vertical mergers cases. In the Figure 8, we depict the situations in which the global risk is greater in the case of conglomerate mergers compared to the case of downstream vertical ones. In fact, conglomerate mergers would have a greater difficulty of integration, which would increase the risks of mergers failure (as studied in Cartwright).

\section{Conclusion}

The technique described in the chapter serves at highlighting the strategic action better suited for reducing the risks in the supply chains. Studying the implications of the supply chain modeling based on the strategic actions like the ones of mergers and acquisitions can improve the quality of mergers suggestions, by highlighting the positive aspects (such as cost reduction as a result of economies of scale) but also the negative aspects (a possible increase in the supply chain's risk) of such a suggested merger.

The results from our studies can be further developed by varying on the construction of the Virtualized Supply Chains set, which should reveal interesting facts on the proposed risk management strategies by applying restructuring activities. The current approach starts with the construction of the VSC from a given business class and it appends business classes 
to the end of the Virtualized Supply Chain until no more supply chains can be appended. In the case of the bounded supply chain, the process stops when the length of the supply chain reaches the threshold. This approach conducts to the computation of the same number of Virtualized Supply Chains in the case of the unbounded VSC experiments and bounded VSC experiments, as the bounded VSCs are sub-Virtualized Supply Chains of the unbounded VSC. A different approach would consists in dividing an $n$-bounded supply chain of the form Class $_{1}$, Class $_{2}, \ldots$, Class $_{n-1}$, Class $_{n}$ in two n-1-bounded VSCs: Class 1, Class $_{2}, \ldots$, Class $_{n-1}$ and Class $_{2}, \ldots$, Class $_{n-1}$, Class $_{n}$.

Examining which merger strategy is better suited for reducing the risks in the Virtualized Supply Chains set constitutes an intermediary step in further developing more advanced techniques for suggesting mergers between two or more business entities.

\section{Acknowledgment}

This work has been supported by CNCSIS-UEFISCSU, under the Project no. 397/2010 "Collaborative Methods for Improving Merger Decisions Support (MECIDEF)".

\section{References}

Bruner, R. F., (2004), Applied Mergers and Acquisitions, John Wiley and Sons, pg. 141.

Cartwright, S., Why Mergers Fail and How To Prevent it, QFinance, http:/ / www.qfinance.com/mergers-and-acquisitions-best-practice/why-mergersfail-and-how-to-prevent-it?full, accessed on 25/03/2011.

DePamphilis, D., (2009), Mergers, Acquisitions and Other Restructuring Activities, Academic Press, pp. 224-225.

Fuld, L. M., (2006), The Secret Language of Competitive Intelligence, Crown Business, pp. 226-227.

Hancu, L., (2008a). Data-Mining Techniques for Supporting Merging Decisions, International Journal of Computers, Communications and Control, Volume:3, Supplementary Issue: ICCCC 2008, pp. 322-326, Oradea, Romania.

Hancu, L., (2008b), Supply Chain Risk Management by Mining Business Dependencies, Proceedings of the International Conference SYNASC, September 2008, Timisoara, Romania.

Hancu, L., (2011), Creating Virtual Communities by Means of Swarm Intelligence, Broad Research in Artificial Intelligence and Neuroscience, Vol. 2, Number 1, pp. 38-43.

Harrigan, K.R., Vertical Integration and Corporate Strategy, Academy of Management Journal, Vol. 28, No. 2, pp. 397-425.

Huo, Y., Jiang., X., Jia, F., and Li, B. (2009), A Framework and Key Techniques for Supply Chain Integration, in Supply Chain - The Way to Flat Organization, Edited by Yanfang Huo and Fu Jia, I-Tech Publishing, Vienna, Austria.

Kiser, J., Cantrell, G., Six Steps to Managing Risk, Supply Chain Management Review, 10(3), pp. 12-17.

Meca, A., Timmer, J., (2008), Supply Chain Collaboration, in Supply Chain - Theory and Applications, Edited by Vedran Kordic, I-Tech Education and Publishing, Croatia, pp. $1-18$.

Preda, G., (2009), Tornado: Merger with RHS brings us back on retail, Business Standard, July 24.

Sliwa, C., (2004), Wal-Mart Takes 'Tough Love' Approach with RFID Directive, Computerworld, January 19, 2004. 


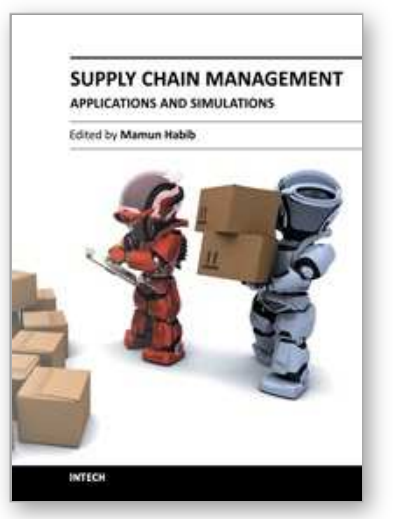

\author{
Supply Chain Management - Applications and Simulations \\ Edited by Prof. Dr. Md. Mamun Habib
}

ISBN 978-953-307-250-0

Hard cover, 252 pages

Publisher InTech

Published online 12, September, 2011

Published in print edition September, 2011

Supply Chain Management (SCM) has been widely researched in numerous application domains during the last decade. Despite the popularity of SCM research and applications, considerable confusion remains as to its meaning. There are several attempts made by researchers and practitioners to appropriately define SCM. Amidst fierce competition in all industries, SCM has gradually been embraced as a proven managerial approach to achieving sustainable profits and growth. This book "Supply Chain Management - Applications and Simulations" is comprised of twelve chapters and has been divided into four sections. Section I contains the introductory chapter that represents theory and evolution of Supply Chain Management. This chapter highlights chronological prospective of SCM in terms of time frame in different areas of manufacturing and service industries. Section II comprised five chapters those are related to strategic and tactical issues in SCM. Section III encompasses four chapters that are relevant to project and technology issues in Supply Chain. Section IV consists of two chapters which are pertinent to risk managements in supply chain.

\title{
How to reference
}

In order to correctly reference this scholarly work, feel free to copy and paste the following:

Lucian Hancu (2011). Supply Chain Modeling Based on Restructuring Activities, Supply Chain Management Applications and Simulations, Prof. Dr. Md. Mamun Habib (Ed.), ISBN: 978-953-307-250-0, InTech, Available from: http://www.intechopen.com/books/supply-chain-management-applications-and-simulations/supply-chainmodeling-based-on-restructuring-activities

\section{INTECH}

open science | open minds

\section{InTech Europe}

University Campus STeP Ri

Slavka Krautzeka 83/A

51000 Rijeka, Croatia

Phone: +385 (51) 770447

Fax: +385 (51) 686166

www.intechopen.com

\section{InTech China}

Unit 405, Office Block, Hotel Equatorial Shanghai

No.65, Yan An Road (West), Shanghai, 200040, China

中国上海市延安西路65号上海国际贵都大饭店办公楼 405 单元

Phone: +86-21-62489820

Fax: $+86-21-62489821$ 
(C) 2011 The Author(s). Licensee IntechOpen. This chapter is distributed under the terms of the Creative Commons Attribution-NonCommercialShareAlike-3.0 License, which permits use, distribution and reproduction for non-commercial purposes, provided the original is properly cited and derivative works building on this content are distributed under the same license. 